\title{
Serial interval of SARS-CoV-2 was shortened over time by nonpharmaceutical interventions
}

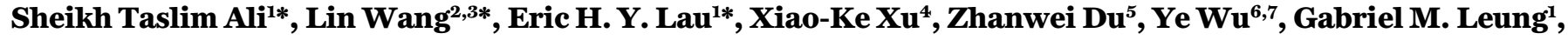 \\ Benjamin J. Cowling'† \\ ${ }^{1}$ WHO Collaborating Centre for Infectious Disease Epidemiology and Control, School of Public Health, Li Ka Shing Faculty of Medicine, The University of Hong Kong, Hong \\ Kong Special Administrative Region, China. ${ }^{2}$ Department of Genetics, University of Cambridge, Cambridge CB2 3EH, UK. ${ }^{3}$ Mathematical Modelling of Infectious Diseases \\ Unit, Institut Pasteur, UMR2000, CNRS, Paris 75015, France. ${ }^{4}$ College of Information and Communication Engineering, Dalian Minzu University, Dalian 116600, China. \\ ${ }^{5}$ Department of Integrative Biology, University of Texas at Austin, Austin, TX 78705, USA. ${ }^{6}$ School of Journalism and Communication, Beijing Normal University, Beijing \\ 100875, China. ${ }^{7}$ Computational Communication Research Center, Beijing Normal University, Zhuhai 519087, China.
}

*These authors contributed equally to this work.

†Corresponding author. Email: bcowling@hku.hk

Studies of novel coronavirus disease (COVID-19) have reported varying estimates of epidemiological parameters including serial interval distributions, i.e., the time between illness onset in successive cases in a transmission chain, and reproduction numbers. By compiling a line-list database of transmission pairs in mainland China, we show that mean serial intervals of COVID-19 have shortened substantially from 7.8 days to 2.6 days within a month (January 9 to February 13,2020). This change is driven by enhanced nonpharmaceutical interventions, in particular case isolation. We also show that using real-time estimation of serial intervals allowing for variation over time, provides more accurate estimates of reproduction numbers than using conventionally fixed serial interval distributions. These findings could improve assessment of transmission dynamics, forecasting future incidence, and estimating the impact of control measures.

In December 2019, a novel coronavirus disease (COVID-19), caused by severe acute respiratory syndrome coronavirus 2 (SARS-CoV-2), was first reported in Wuhan, China, and has since spread to more than 212 countries, causing more than 10 million confirmed cases and 500,000 deaths worldwide by 30 June 2020 (1). Recent studies suggest that several demographic and social factors can influence the transmission of COVID-19, including age and gender-related difference in infection risk (2-4), reduced risk of infection as a result of intensive non-pharmaceutical interventions (NPIs) (e.g., isolation, social distancing) (5-7), and abrupt changes in social mixing patterns because of lockdowns and confinement (8-10). Serial interval, defined as the duration between the symptom onset time of infector and that of the infectee, is an essential metric for estimating many other key epidemiological parameters (e.g., reproduction number, generation time, and attack rate), which are in turn used to predict disease trends and healthcare demands (11). In early studies before availability of specific data on COVID-19, the serial interval distribution of COVID-19 was assumed to be similar to that of Severe Acute Respiratory Syndrome or Middle East Respiratory Syndrome, with a mean $>8$ days (12, 13). Once specific data became available on COVID-19 transmission pairs, several studies have examined the serial interval distribution of COVID-19 in different locations, with estimates of the mean serial interval varying from 3.1 days to 7.5 days $(6,14-21)$. All these studies assumed that the timing of transmission events can be described by a single stable distribution of serial intervals at different stage of an epidemic.

In fact, the serial interval depends on the incubation period and the profile of infectiousness after infection as well as the variation in contact structure of the population (as explained in fig. S1) (22). The incubation period describes the biological process of disease progression and tends to follow a more similar distribution from one location to another, with minor differences resulting from social or cultural differences in how symptoms are perceived or reported. However, the profile of infectiousness over time can vary because of human behavior. Changes in contact patterns and the use of public health measures can reshape the timing of infection events by limiting successful contacts overall (e.g., social distancing) or after illness onset (e.g., case isolation). Interventions, such as the isolation of confirmed and suspected cases, suspension of intra- and inter-city travel, and different forms of social distancing were widely implemented in different Chinese cities. This provides an opportunity to study the temporal changes in the serial interval distribution and its association with NPIs. Here, we show that variation in the serial interval can occur and has important implications for the 
assessment of transmission dynamics and the impact of control measures.

We compiled a database of 1,407 COVID-19 transmission pairs, in which symptom onset dates and social relationships were available for both the infector and infectee of 677 transmission pairs (see table S1 for entire database (23) and supplementary materials). Household and non-household transmissions were identified from the information on social relationships (e.g., familial members of the same household, non-household relatives, colleagues, classmates, friends, and other face-to-face contacts). The data were reconstructed from the publicly available reports of 9,120 confirmed COVID-19 cases reported by 27 provincial and 264 urban health commissions in China outside Hubei province. Data from Hubei province were excluded because there was less reliable information on chains of transmission during the widespread community circulation of COVID-19, whereas outside Hubei province it was more straightforward to link connected cases and derive serial intervals. We focused on 677 transmission pairs with infectors having developed symptoms from January 9 through February 13, 2020. This 36-day period covers a series of key interventions related to the evolving epidemiology and transmission dynamics of COVID19 in mainland China (24-26).

We first calculated the number of transmission pairs in our database by the onset dates of infectors (fig. S3). Since many infectors (339) developed symptoms during January 23-29, 2020, we defined this 1-week period as the peak-week, the previous 14-day period (January 9-22, 2020) as the prepeak period, and the following 15-day period (January 30 February 13, 2020) as the post-peak period. We computed the serial interval as the number of days between the symptom onset dates of the infector and the infectee for each transmission pair. Empirical serial interval distributions for transmission pairs counting from symptom onsets of the infectors during each period indicate that the serial intervals shortened over time (Fig. 1A).

We estimated the serial interval distribution during each non-overlapping period by fitting a normal distribution to the corresponding serial intervals data (supplementary materials). Analysis of all 677 transmission pairs revealed that the serial interval distribution had a mean of 5.1 (95\% credibility interval, CrI: 4.7, 5.5) days and standard deviation of 5.3 (95\% CrI: 5.0, 5.6) days (table S2) overall, consistent with other recent studies $(16,21,27)$. However, fitting to data of non-overlapping periods of time revealed considerable variation in serial interval distributions (Fig. 1B). Prior to the peak, the mean and standard deviation of serial intervals were estimated to be $7.8(7.0,8.6)$ days and $5.2(4.7,5.9)$ days. During the peak, the mean and standard deviation reduced to 5.1 $(4.6,5.7)$ days and $5.0(4.6,5.4)$ days, respectively. After the peak, these estimates further shortened to 2.6 (1.9, 3.2) days and $4.6(4.2,5.1)$ days, respectively (table S2).

Next, we examined the real-time change in serial intervals by using a series of running time windows with fixed length of 10,14 or 18 days (fig. S10). In contrast to the use of a constant distribution of serial intervals, our analysis suggests that serial intervals were gradually shortened over the study period (Fig. 2A), which is robust to alternative specifications of time windows (fig. S10). By fitting the transmission pairs data for each running time-window by Markov Chain Monte Carlo (MCMC) (Fig. 2A and table S3), we estimated that during the first 14-day period (January $9-22,2020$ ) the serial intervals were longer on average (mean: 7.8 (95\% CrI: 7.0, 8.6) days, and standard deviation (sd): 5.2 (95\% CrI: 4.7, 5.9) days), whereas during the last 14 days (January 30 - February $13,2020)$ the serial intervals were much shorter on average (mean: $2.2(1.5,2.9)$ days, and sd: $4.6(4.1,5.1)$ days). Notably, the mean serial intervals shortened by more than threefold over the 36-day period.

The transmission pair data also contains information for age, sex, household, and isolation delay (i.e., time duration from symptom onset to isolation) for most infectors. This allows a granular stratification. Using either non-overlapping or running time windows for data stratified by each of these factors, we find the same pattern of shorter serial intervals over time (Figs. 1B and 2A and tables S2 and S3). Therefore, we termed this changing serial interval the "effective serial interval", which accounts for temporal changes caused by its potential driving factors. Notably, the length of effective serial intervals is positively associated with the length of isolation delay (Fig. 2A; figs. S5, S6, and S9; and tables S3 and S4), accounting for the decreasing isolation delay over time (fig. S2). Therefore, early isolation (shorter than the median isolation delay) translates into shorter serial intervals (mean: 3.3 $(2.7,3.8)$ days, and sd: $4.5(4.1,4.9)$ days), and delayed isolation (longer than the median isolation delay) is associated with a longer serial interval (mean: $6.8(6.2,7.3)$ days, and sd: $5.3(4.9,5.7)$ days) (table S2). Stratification by age, gender or household shows no clear difference in serial interval estimates. Our findings are robust to using alterative distributions (e.g., Gumbel distribution) for model fitting (fig. S11) and the infector-based approach (fig. S14).

Our probabilistic, individual-based simulated and regression models confirm that serial intervals are positively associated with isolation delay (section 5, supplementary materials). We found that the serial interval become shorter based on how faster the infectors are isolated, regardless of when an infector starts to be infectious before illness onset (fig. S5). In an individual-based simulation model with a mean generation time of 7.8 days, the simulated mean serial intervals reduces from $\sim 8.0$ to $\sim 1.2$ days when the isolation delay reduces from 10 to 0 days. We found up to $51.5 \%$ of the variability in daily empirical serial interval can be explained 
by isolation delay through regression model and further improved by other NPI factors, explaining additional $15.6 \%$ to $16.7 \%$ of the variability (table S5).

In practice, the time varying serial interval may affect the estimation of epidemic parameters including the transmissibility. The real-time transmissibility of an infectious disease is often characterized by the instantaneous reproduction number $\left(R_{t}\right)$, which is defined as the expected number of secondary infections caused by an infector on day $t$. The pathogen spreads when $R_{t}>1$ and is under control when $R_{t}<1$. To examine the effect of serial intervals on $R_{t}$, we first obtained the daily number of cases based on the onset dates of infectors and infectees among the 1,407 transmission pairs (Fig. 2, B to D). By applying the statistical method developed by Cori et al. (28), we estimated $R_{t}$ for each day between January 20 and February 13, 2020. We noticed substantial differences in estimates of $R_{t}$ between using a single stable serial interval distribution and time-varying effective serial interval distributions. The magnitude of this difference is more prominent during the pre-peak and post-peak periods compared to that of during the peak week when $R_{t} \approx 1$ (Fig. 2, B to D).

We observed that the serial interval for COVID-19 in mainland China shortened more than threefold in the 36days between January 9 - February 13, 2020. This reduction was driven by intensive non-pharmaceutical interventions, particularly, reduction of the isolation delay period. Isolation of an infector one day earlier is expected to reduce the mean serial interval by 0.7 days. Thus, the serial interval was shortened by $>3$ days if infectors were rapidly isolated (Figs. 1B and $2 \mathrm{~A}$ and tables $\mathrm{S} 2$ and $\mathrm{S} 3$ ). This is consistent with advocating isolation of cases and quarantining contacts within a day from symptom onset, which has been estimated to reduce COVID-19 transmission by $60 \%$ (8). We have not identified any significant effects of gender or age of infectors, on serial interval, but the NPIs were found to be significant for the transmission in community rather than in households (table S5). Other studies $(15,20)$ have estimated that the transmissibility of COVID-19 is greater at symptom onset. Although a short serial interval indicates that a substantial proportion of transmission events have occurred by the time symptoms are apparent (14), because of prolonged viral shedding (14, 29, 30 ) case isolation is still likely to reduce further transmission. Changes in the serial interval can therefore indicate effective implementation of specific transmission reduction measures.

Limitations of our work: First, possible recall bias on the onset of first symptoms in the line-list data; however, given the centralized pandemic response in mainland China, we expected that recall bias would not affect our main conclusions (figs. S12 and S13). Second, other factors may have influenced the reduction of effective serial intervals as we can only explain up to $72 \%$ of the variance in observed serial intervals. Finally, our current transmission pair data did not contain variables about potential exposure window of each case, which did not allow further inferences on the transmission potential.

Our results indicate that caution is needed when attempting to generalize estimates of the serial interval distribution to other places or to other periods in the same place, for example when estimating instantaneous reproductive numbers (Fig. 2, B to D). The real-time metric of effective serial intervals indicates that transmission models also need to account for temporal variation in serial intervals as epidemic proceeds. Effective serial intervals may provide better measurement of instantaneous transmissibility $\left(R_{t}\right)$ because they include the effects of possible drivers of transmission, and would be helpful to policy makers because they offer realtime information on the impact of public health measures.

\section{REFERENCES AND NOTES}

1. World Health Organization (WHO), "Coronavirus disease 2019 (COVID-19): Situation report - 162" (WHO, 2020); www.who.int/docs/defaultsource/coronaviruse/situation-reports/20200629-covid-19-sitrep161.pdf?sfvrsn=74fde64e_2).

2. M. U. G. Kraemer, C.-H. Yang, B. Gutierrez, C.-H. Wu, B. Klein, D. M. Pigott, Open COVID-19 Data Working Group, L. du Plessis, N. R. Faria, R. Li, W. P. Hanage, J. S. Brownstein, M. Layan, A. Vespignani, H. Tian, C. Dye, O. G. Pybus, S. V. Scarpino, The effect of human mobility and control measures on the COVID-19 epidemic in China. Science 368, 493-497 (2020). doi:10.1126/science.abb4218 Medline

3. C. Wenham, J. Smith, R. Morgan, Gender and COVID-19 Working Group, COVID-19: The gendered impacts of the outbreak. Lancet 395, 846-848 (2020). doi:10.1016/S0140-6736(20)30526-2 Medline

4. J. M. Jin, P. Bai, W. He, F. Wu, X.-F. Liu, D.-M. Han, S. Liu, J.-K. Yang, Gender Differences in Patients With COVID-19: Focus on Severity and Mortality. Front. Public Health 8, 152 (2020). doi:10.3389/fpubh.2020.00152 Medline

5. J. Hellewell, S. Abbott, A. Gimma, N. I. Bosse, C. I. Jarvis, T. W. Russell, J. D. Munday, A. J. Kucharski, W. J. Edmunds, Centre for the Mathematical Modelling of Infectious Diseases COVID-19 Working Group, S. Funk, R. M. Eggo, Feasibility of controlling COVID-19 outbreaks by isolation of cases and contacts. Lancet Glob. Health 8, e488-e496 (2020). doi:10.1016/S2214-109X(20)30074-7 Medline

6. L. Ferretti, C. Wymant, M. Kendall, L. Zhao, A. Nurtay, L. Abeler-Dörner, M. Parker, D. Bonsall, C. Fraser, Quantifying SARS-CoV-2 transmission suggests epidemic control with digital contact tracing. Science 368, eabb6936 (2020). doi:10.1126/science.abb6936 Medline

7. R. Armitage, L. B. Nellums, COVID-19 and the consequences of isolating the elderly. Lancet Public Health 5, e256 (2020). doi:10.1016/S2468-2667(20)30061-X Medline

8. R. M. Anderson, H. Heesterbeek, D. Klinkenberg, T. D. Hollingsworth, How will country-based mitigation measures influence the course of the COVID-19 epidemic? Lancet 395, 931-934 (2020). doi:10.1016/S0140-6736(20)30567-5 Medline

9. J. R. Koo, A. R. Cook, M. Park, Y. Sun, H. Sun, J. T. Lim, C. Tam, B. L. Dickens, Interventions to mitigate early spread of SARS-CoV-2 in Singapore: A modelling study. Lancet Infect. Dis. 20, 678-688 (2020). doi:10.1016/S14733099(20)30162-6 Medline

10. K. Prem, Y. Liu, T. W. Russell, A. J. Kucharski, R. M. Eggo, N. Davies, Centre for the Mathematical Modelling of Infectious Diseases COVID-19 Working Group, M. Jit, P. Klepac, The effect of control strategies to reduce social mixing on outcomes of the COVID-19 epidemic in Wuhan, China: A modelling study. Lancet Public Health 5, e261-e270 (2020). doi:10.1016/S2468-2667(20)30073-6 Medline

11. M. A. Vink, M. C. Bootsma, J. Wallinga, Serial intervals of respiratory infectious diseases: A systematic review and analysis. Am. J. Epidemiol. 180, 865-875 (2014). doi:10.1093/aje/kwu209 Medline 
12. J. T. Wu, K. Leung, G. M. Leung, Nowcasting and forecasting the potential domestic and international spread of the 2019-nCoV outbreak originating in Wuhan, China: A modelling study. Lancet 395, 689-697 (2020). doi:10.1016/S01406736(20)30260-9 Medline

13. M. Chinazzi, J. T. Davis, M. Ajelli, C. Gioannini, M. Litvinova, S. Merler, A. Pastore Y Piontti, K. Mu, L. Rossi, K. Sun, C. Viboud, X. Xiong, H. Yu, M. E. Halloran, I. M. Longini Jr., A. Vespignani, The effect of travel restrictions on the spread of the 2019 novel coronavirus (COVID-19) outbreak. Science 368, 395-400 (2020). doi:10.1126/science.aba9757 Medline

14. H. Nishiura, N. M. Linton, A. R. Akhmetzhanov, Serial interval of novel coronavirus (COVID-19) infections. Int. J. Infect. Dis. 93, 284-286 (2020). doi:10.1016/i.iiid.2020.02.060 Medline

15. H. Y. Cheng, S.-W. Jian, D.-P. Liu, T.-C. Ng, W.-T. Huang, H.-H. Lin, Taiwan COVID19 Outbreak Investigation Team, Contact Tracing Assessment of COVID-19 Transmission Dynamics in Taiwan and Risk at Different Exposure Periods Before and After Symptom Onset. JAMA Intern. Med. 10.1001/jamainternmed.2020.2020 doi:10.1001/iamainternmed.2020.2020 Medline

16. Z. Du, X. Xu, Y. Wu, L. Wang, B. J. Cowling, L. A. Meyers, Serial Interval of COVID19 among Publicly Reported Confirmed Cases. Emerg. Infect. Dis. 26, 1341-1343 (2020). doi:10.3201/eid2606.200357 Medline

17. Q. Li, X. Guan, P. Wu, X. Wang, L. Zhou, Y. Tong, R. Ren, K. S. M. Leung, E. H. Y. Lau, J. Y. Wong, X. Xing, N. Xiang, Y. Wu, C. Li, Q. Chen, D. Li, T. Liu, J. Zhao, M. Liu, W. Tu, C. Chen, L. Jin, R. Yang, Q. Wang, S. Zhou, R. Wang, H. Liu, Y. Luo, Y. Liu, G. Shao, H. Li, Z. Tao, Y. Yang, Z. Deng, B. Liu, Z. Ma, Y. Zhang, G. Shi, T. T. Y. Lam, J. T. Wu, G. F. Gao, B. J. Cowling, B. Yang, G. M. Leung, Z. Feng, Early Transmission Dynamics in Wuhan, China, of Novel Coronavirus-Infected Pneumonia. N. Engl. J. Med. 382, 1199-1207 (2020). doi:10.1056/NEJMoa2001316 Medline

18. J. M. Griffin, A. B. Collins, K. Hunt, D. McEvoy, M. Casey, A. W. Byrne, C. G. McAloon, A. Barber, E. A. Lane, S. J. More, A rapid review of available evidence on the serial interval and generation time of COVID-19. medRxiv 2020.05.08.20095075 [Preprint]. 11 May 2020. https://doi.org/10.1101/2020.05.08.20095075.

19. S. Ma, J. Zhang, M. Zeng, Q. Yun, W. Guo, Y. Zheng, S. Zhao, M. H. Wang, Z. Yang, Epidemiological parameters of coronavirus disease 2019: A pooled analysis of publicly reported individual data of 1155 cases from seven countries. medRxiv 2020.03.21.20040329 [Preprint]. 24 March 2020. https://doi.org/10.1101/2020.03.21.20040329.

20. X. He, E. H. Y. Lau, P. Wu, X. Deng, J. Wang, X. Hao, Y. C. Lau, J. Y. Wong, Y. Guan, X. Tan, X. Mo, Y. Chen, B. Liao, W. Chen, F. Hu, Q. Zhang, M. Zhong, Y. Wu, L. Zhao, F. Zhang, B. J. Cowling, F. Li, G. M. Leung, Temporal dynamics in viral shedding and transmissibility of COVID-19. Nat. Med. 26, 672-675 (2020). doi:10.1038/s41591-020-0869-5 Medline

21. Q. Bi, Y. Wu, S. Mei, C. Ye, X. Zou, Z. Zhang, X. Liu, L. Wei, S. A. Truelove, T. Zhang, W. Gao, C. Cheng, X. Tang, X. Wu, Y. Wu, B. Sun, S. Huang, Y. Sun, J. Zhang, T. Ma, J. Lessler, T. Feng, Epidemiology and transmission of COVID-19 in 391 cases and 1286 of their close contacts in Shenzhen, China: A retrospective cohort study. Lancet Infect. Dis. 10.1016/S1473-3099(20)30287-5 (2020). doi:10.1016/S14733099(20)30287-5 Medline

22. J. Zhang, M. Litvinova, Y. Liang, Y. Wang, W. Wang, S. Zhao, Q. Wu, S. Merler, C. Viboud, A. Vespignani, M. Ajelli, H. Yu, Changes in contact patterns shape the dynamics of the COVID-19 outbreak in China. Science 368, 1481-1486 (2020). doi:10.1126/science.abb8001 Medline

23. Lin, PDGLin/COVID19_EffSeriallnterval_NPI: Serial interval of SARS-CoV-2 was shortened over time by non-pharmaceutical interventions, version v1.0, Zenodo (2020); http://doi.org/10.5281/zenodo.3940300.

24. H. Tian, Y. Liu, Y. Li, C.-H. Wu, B. Chen, M. U. G. Kraemer, B. Li, J. Cai, B. Xu, Q. Yang, B. Wang, P. Yang, Y. Cui, Y. Song, P. Zheng, Q. Wang, O. N. Bjornstad, R. Yang, B. T. Grenfell, O. G. Pybus, C. Dye, An investigation of transmission control measures during the first 50 days of the COVID-19 epidemic in China. Science 368, 638-642 (2020). doi:10.1126/science. abb6105 Medline

25. K. Leung, J. T. Wu, D. Liu, G. M. Leung, First-wave COVID-19 transmissibility and severity in China outside Hubei after control measures, and second-wave scenario planning: A modelling impact assessment. Lancet 395, 1382-1393 (2020). doi:10.1016/S0140-6736(20)30746-7 Medline

26. A. Pan, L. Liu, C. Wang, H. Guo, X. Hao, Q. Wang, J. Huang, N. He, H. Yu, X. Lin, S.
Wei, T. Wu, Association of Public Health Interventions With the Epidemiology of the COVID-19 Outbreak in Wuhan, China. JAMA 323, 1915-1923 (2020). doi:10.1001/iama.2020.6130 Medline

27. J. Zhang, M. Litvinova, W. Wang, Y. Wang, X. Deng, X. Chen, M. Li, W. Zheng, L. Yi, X. Chen, Q. Wu, Y. Liang, X. Wang, J. Yang, K. Sun, I. M. Longini Jr., M. E. Halloran, P. Wu, B. J. Cowling, S. Merler, C. Viboud, A. Vespignani, M. Ajelli, H. Yu, Evolving epidemiology and transmission dynamics of coronavirus disease 2019 outside Hubei province, China: A descriptive and modelling study. Lancet Infect. Dis. 20 793-802 (2020). doi:10.1016/S1473-3099(20)30230-9 Medline

28. A. Cori, N. M. Ferguson, C. Fraser, S. Cauchemez, A new framework and software to estimate time-varying reproduction numbers during epidemics. Am. J. Epidemiol. 178, 1505-1512 (2013). doi:10.1093/aje/kwt133 Medline

29. L. Zou, F. Ruan, M. Huang, L. Liang, H. Huang, Z. Hong, J. Yu, M. Kang, Y. Song, J. Xia, Q. Guo, T. Song, J. He, H.-L. Yen, M. Peiris, J. Wu, SARS-CoV-2 Viral Load in Upper Respiratory Specimens of Infected Patients. N. Engl. J. Med. 382, 11771179 (2020). doi:10.1056/NEJMc2001737 Medline

30. Y. Pan, D. Zhang, P. Yang, L. L. M. Poon, Q. Wang, Viral load of SARS-CoV-2 in clinical samples. Lancet Infect. Dis. 20, 411-412 (2020). doi:10.1016/S14733099(20)30113-4 Medline

31. X. K. Xu, X.-F. Liu, Y. Wu, S. T. Ali, Z. Du, P. Bosetti, E. H. Y. Lau, B. J. Cowling, L. Wang, Reconstruction of Transmission Pairs for novel Coronavirus Disease 2019 (COVID-19) in mainland China: Estimation of Super-spreading Events, Serial Interval, and Hazard of Infection. Clin. Infect. Dis. ciaa790 (2020). doi:10.1093/cid/ciaa790 Medline

32. P. Trapman, F. Ball, J.-S. Dhersin, V. C. Tran, J. Wallinga, T. Britton, Inferring RO in emerging epidemics-the effect of common population structure is small. J. R. Soc. Interface 13, 20160288 (2016). doi:10.1098/rsif.2016.0288 Medline

33. S. W. Park, D. Champredon, J. Dushoff, Inferring generation-interval distributions from contact-tracing data. J. R. Soc. Interface 17, 20190719 (2020). doi:10.1098/rsif.2019.0719 Medline

34. Q. H. Liu, M. Ajelli, A. Aleta, S. Merler, Y. Moreno, A. Vespignani, Measurability of the epidemic reproduction number in data-driven contact networks. Proc. Natl. Acad. Sci. U.S.A. 115, 12680-12685 (2018). doi:10.1073/pnas.1811115115 Medline

35. Y. Bai, L. Yao, T. Wei, F. Tian, D.-Y. Jin, L. Chen, M. Wang, Presumed Asymptomatic Carrier Transmission of COVID-19. JAMA 323, 1406-1407 (2020). doi:10.1001/jama.2020.2565 Medline

36. X. Pan, D. Chen, Y. Xia, X. Wu, T. Li, X. Ou, L. Zhou, J. Liu, Asymptomatic cases in a family cluster with SARS-CoV-2 infection. Lancet Infect. Dis. 20, 410-411 (2020). doi:10.1016/S1473-3099(20)30114-6 Medline

37. M. M. Arons, K. M. Hatfield, S. C. Reddy, A. Kimball, A. James, J. R. Jacobs, J. Taylor, K. Spicer, A. C. Bardossy, L. P. Oakley, S. Tanwar, J. W. Dyal, J. Harney, Z. Chisty, J. M. Bell, M. Methner, P. Paul, C. M. Carlson, H. P. McLaughlin, N. Thornburg, S. Tong, A. Tamin, Y. Tao, A. Uehara, J. Harcourt, S. Clark, C. Brostrom-Smith, L. C. Page, M. Kay, J. Lewis, P. Montgomery, N. D. Stone, T. A. Clark, M. A. Honein, J. S. Duchin, J. A. Jernigan, Public Health-Seattle and King County and CDC COVID-19 Investigation Team, Presymptomatic SARS-CoV-2 Infections and Transmission in a Skilled Nursing Facility. N. Engl. J. Med. 382, 2081-2090 (2020). doi:10.1056/NEJMoa2008457 Medline

38. T. Ganyani, C. Kremer, D. Chen, A. Torneri, C. Faes, J. Wallinga, N. Hens, Estimating the generation interval for coronavirus disease (COVID-19) based on symptom onset data, March 2020. Euro Surveill. 25, 2000257 (2020). doi:10.2807/15607917.ES.2020.25.17.2000257 Medline

39. W. E. Wei, Z. Li, C. J. Chiew, S. E. Yong, M. P. Toh, V. J. Lee, Presymptomatic Transmission of SARS-CoV-2 - Singapore, January 23-March 16, 2020. MMWR Morb. Mortal. Wkly. Rep. 69, 411-415 (2020). doi:10.15585/mmwr.mm6914e1 Medline

40. L. Tindale, M. Coombe, J. E. Stockdale, E. Garlock, W. Y. V. Lau, M. Saraswat, Y.-H. B. Lee, L. Zhang, D. Chen, J. Wallinga, C. Colijn, Transmission interval estimates suggest pre-symptomatic spread of COVID-19. medRxiv 2020.03.03.20029983 [Preprint]. 6 March 2020. https://doi.org/10.1101/2020.03.03.20029983.

41. R. Wölfel, V. M. Corman, W. Guggemos, M. Seilmaier, S. Zange, M. A. Müller, D. Niemeyer, T. C. Jones, P. Vollmar, C. Rothe, M. Hoelscher, T. Bleicker, S. Brünink, J. Schneider, R. Ehmann, K. Zwirglmaier, C. Drosten, C. Wendtner, Virological assessment of hospitalized patients with COVID-2019. Nature 581, 465-469 (2020). doi:10.1038/s41586-020-2196-x Medline 
42. A. T. Huang, B. Garcia-Carreras, M. D. T. Hitchings, B. Yang, L. Katzelnick, S. M. Rattigan, B. Borgert, C. Moreno, B. D. Solomon, I. Rodriguez-Barraquer, J. Lessler, H. Salje, D. S. Burke, A. Wesolowski, D. A. T. Cummings, A systematic review of antibody mediated immunity to coronaviruses: Antibody kinetics, correlates of protection, and association of antibody responses with severity of disease. medRxiv 2020.04.14.20065771 [Preprint]. 17 April 2020. https://doi.org/10.1101/2020.04.14.20065771.

43. B. Rockx, T. Kuiken, S. Herfst, T. Bestebroer, M. M. Lamers, B. B. Oude Munnink, D. de Meulder, G. van Amerongen, J. van den Brand, N. M. A. Okba, D. Schipper, P. van Run, L. Leijten, R. Sikkema, E. Verschoor, B. Verstrepen, W. Bogers, J. Langermans, C. Drosten, M. Fentener van Vlissingen, R. Fouchier, R. de Swart, M. Koopmans, B. L. Haagmans, Comparative pathogenesis of COVID-19, MERS, and SARS in a nonhuman primate model. Science 368, 1012-1015 (2020). doi:10.1126/science. abb7314 Medline

44. M. Lipsitch, T. Cohen, B. Cooper, J. M. Robins, S. Ma, L. James, G. Gopalakrishna, S. K. Chew, C. C. Tan, M. H. Samore, D. Fisman, M. Murray, Transmission dynamics and control of severe acute respiratory syndrome. Science 300, 1966-1970 (2003). doi:10.1126/science.1086616 Medline

45. K. M. Gostic, L. McGough, E. Baskerville, S. Abbott, K. Joshi, C. Tedijanto, R. Kahn, R. Niehus, J. A. Hay, P. M. De Salazar, J. Hellewell, S. Meakin, J. Munday, N. Bosse, K. Sherratt, R. M. Thompson, L. F. White, J. Huisman, J. Scire, S. Bonhoeffer, T. Stadler, J. Wallinga, S. Funk, M. Lipsitch, S. Cobey, Practical considerations for measuring the effective reproductive number, Rt. medRxiv 2020.06.18.20134858 [Preprint]. 23 June 2020. https://doi.org/10.1101/2020.06.18.20134858.

46. J. Wallinga, P. Teunis, Different epidemic curves for severe acute respiratory syndrome reveal similar impacts of control measures. Am. J. Epidemiol. 160, 509-516 (2004). doi:10.1093/aje/kwh255 Medline

47. C. Fraser, Estimating individual and household reproduction numbers in an emerging epidemic. PLOS ONE 2, e758 (2007). doi:10.1371/journal.pone.0000758 Medline

48. Z. Li, Q. Chen, L. Feng, L. Rodewald, Y. Xia, H. Yu, R. Zhang, Z. An, W. Yin, W. Chen, Y. Qin, Z. Peng, T. Zhang, D. Ni, J. Cui, Q. Wang, X. Yang, M. Zhang, X. Ren, D. Wu, X. Sun, Y. Li, L. Zhou, X. Qi, T. Song, G. F. Gao, Z. Feng, China CDC COVID-19 Emergency Response Strategy Team, Active case finding with case management: The key to tackling the COVID-19 pandemic. Lancet 396, 63-70 (2020). doi:10.1016/S0140-6736(20)31278-2 Medline

49. P. Wu, X. Hao, E. H. Y. Lau, J. Y. Wong, K. S. M. Leung, J. T. Wu, B. J. Cowling, G. M. Leung, Real-time tentative assessment of the epidemiological characteristics of novel coronavirus infections in Wuhan, China, as at 22 January 2020. Euro Surveill. 25, 2000044 (2020). doi:10.2807/1560-7917.ES.2020.25.3.2000044 Medline

\section{ACKNOWLEDGMENTS}

We thank all health workers and volunteers who collected data throughout the COVID-19 outbreak. We thank Henrik Salje, Simon Cauchemez, Lauren Ancel Meyers, Juliette Paireau, Qifang Bi, Bingyi Yang, Xiaofan Liu, and Lanfang Hu for discussions. Funding: We acknowledge financial support from the Health and Medical Research Fund, Food and Health Bureau, Government of the Hong Kong Special Administrative Region, China (grant no. COVID190118), Investissement d'Avenir program, the Laboratoire d'Excellence Integrative Biology of Emerging Infectious Diseases program (grant no. ANR-10-LABX-62-IBEID), European Research Council (grant no. 804744), European Union's Horizon 2020 research and innovation program under grant agreement no. 101003589, National Institutes of Health (U01 GM087719), Open Fund of Key Laboratory of Urban Land Resources Monitoring and Simulation, Ministry of Land and Resources (KF2019-04-034), National Natural Science Foundation of China (61773091, 11875005, 61976025, 11975025), and University of Cambridge COVID-19 Rapid Response Grant. Author contributions: L.W., S.T.A., E.H.Y.L, and B.J.C.: conceived the study, designed statistical and modeling methods, conducted analyses, interpreted results, wrote and revised the manuscript; X.-K.X., Z. Du, and Y.W.: collected and compiled data, interpreted results, and revised the manuscript; G.M.L. supervised the study, interpreted results, and revised the manuscript. Competing interests: BJC reports honoraria from Sanofi Pasteur and Roche. The authors report no other potential conflicts of interest. Data and materials availability: All data and codes are available in the main text or the supplementary materials or in reference (23). This work is licensed under a Creative Commons Attribution 4.0 International (CC BY 4.0) license, which permits unrestricted use, distribution, and reproduction in any medium, provided the original work is properly cited. To view a copy of this license, visit https:/creativecommons.org/licenses/by/4.0/. This license does not apply to figures/photos/artwork or other content included in the article that is credited to a third party; obtain authorization from the rights holder before using such material.

\section{SUPPLEMENTARY MATERIALS}

science.sciencemag.org/cgi/content/full/science.abc9004/DC1

Materials and Methods

Figs. S1 to S14

Tables S1 to S5

References (31-49)

MDAR Reproducibility Checklist

20 May 2020; accepted 13 July 2020

Published online 21 July 2020

10.1126/science.abc9004 

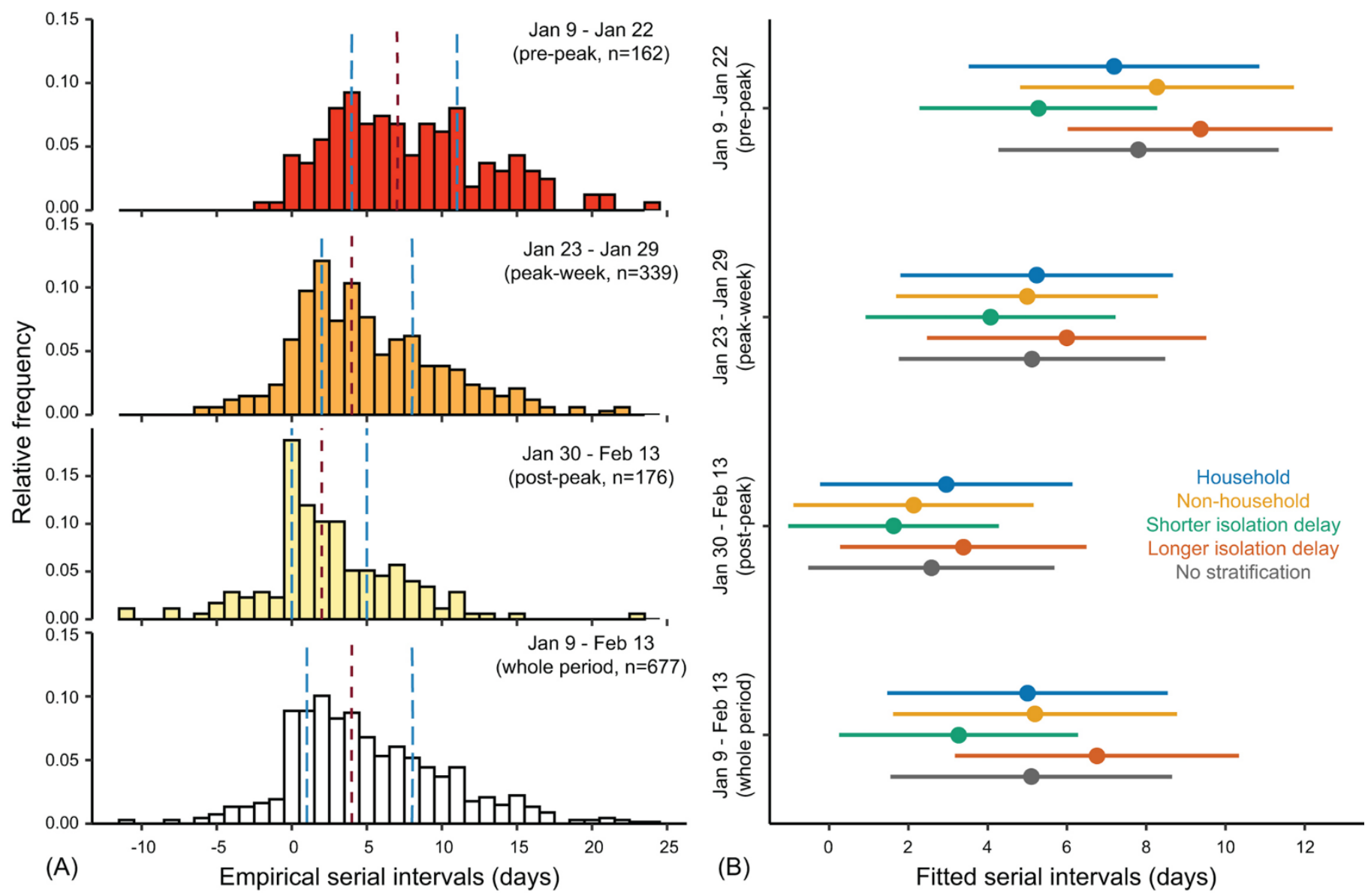

Fig. 1. Serial intervals of SARS-CoV-2 substantially shortened over time in mainland China. (A) Empirical serial interval distributions. From top to bottom, transmission pairs were analyzed by selecting infectors who developed symptoms during January 9 _ 22, 2020 (pre-peak), January 23 _ 29, 2020 (peak-week), January 30 _ February 13, 2020 (post-peak), and January 9 _ February 13, 2020 (whole period), respectively. In each panel, vertical dashed lines in red and blue colors indicate the median and interquartile range (IQR). (B) Estimated serial interval distributions by fitting a normal distribution via MCMC. From top to bottom, each group of bars correspond to the transmission pairs with infectors who developed symptom during the pre-peak (162 pairs), peak-week (339 pairs), post-peak (176 pairs), and whole 36-day period, respectively. Colored dots and bars correspond to the transmission pairs within households (blue), outside households (yellow), with isolation delay shorter than the median isolation delay of each period (green), and with isolation delay longer than the median isolation delay of each period (orange), respectively. Dark-grey bars correspond to transmission pairs with no stratification. Dots and bars indicate the estimated median and IQR, respectively. 

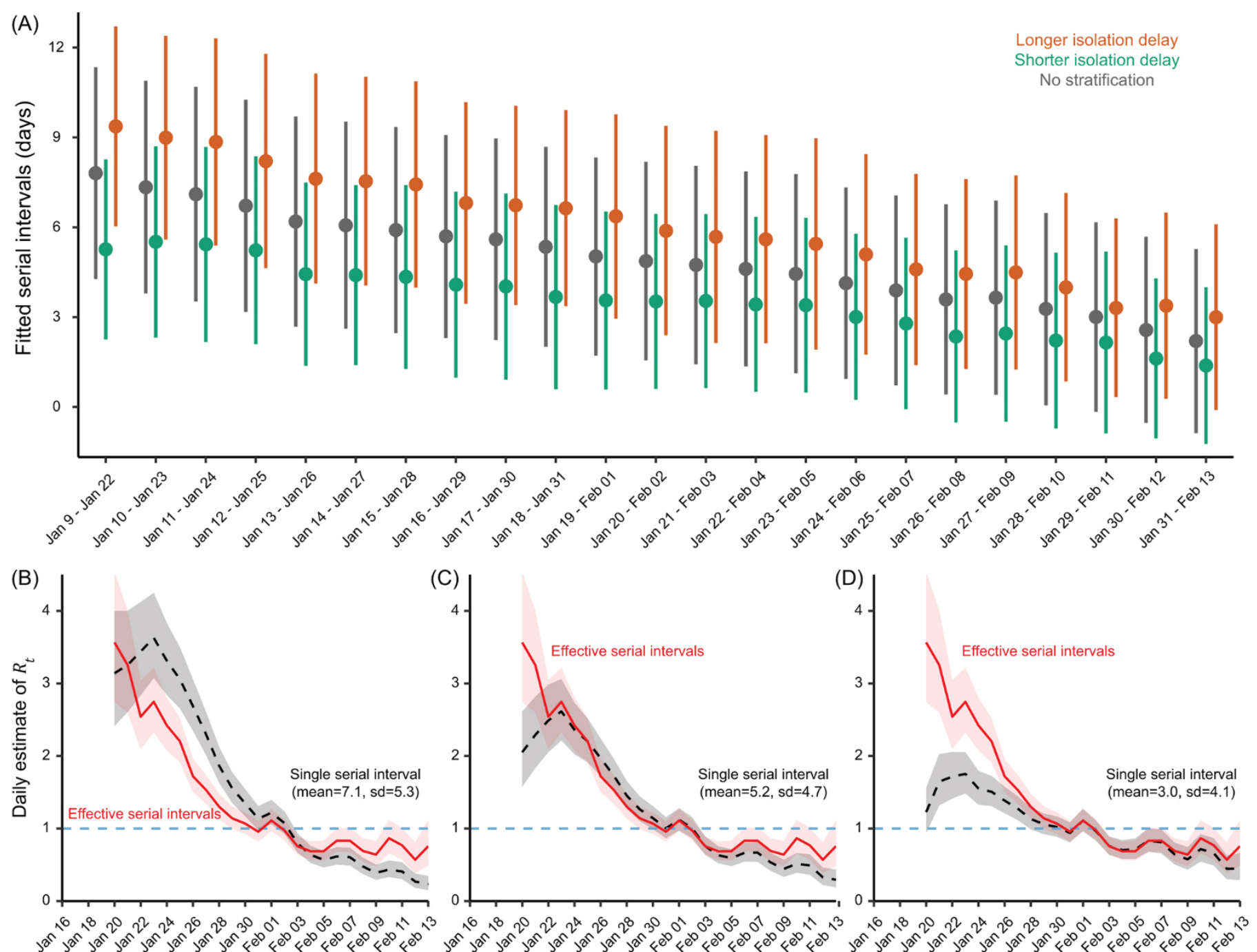

Fig. 2. Real-time effective serial intervals and instantaneous reproduction number $R_{t .}(\mathrm{A})$ Estimated serial interval distribution for each 14-day running time window. Dark-grey color indicates fitting data with no stratification, whereas green (yellow) indicates fitting data with isolation delay shorter (longer) than the median isolation delay of each running time window. Dots and bars indicate the estimated median and IQR, respectively. ( $\mathrm{B}$ to $\mathrm{D}$ ) Daily estimates of $R_{t}$ by using real-time effective serial interval distributions (as panel $(\mathrm{A})$ ) versus using a single fixed serial interval distribution. Red curves and light-pink shaded regions indicate the median and 95\% $\mathrm{Crl}$ of daily $R_{t}$ estimated using real-time effective serial interval distributions. Black dashed curves and light-grey shaded regions indicate the median and $95 \% \mathrm{Crl}$ of daily $R_{t}$ estimated using a single serial interval distribution fixed with (B) mean=7.1, $s d=5.3$ days, $(C)$ mean=5.2, $s d=4.7$ days, and (D) mean=3.0, $s d=4.1$ days. 